\title{
INDEX OF VOLUME XXVI.
}

Alexander, J. W. Note on Riemann Spaces, 370.

Allen, F. E. See Reviews, under Wentworth.

Altshiller-Court, N. On a Pencil of Nodal Cubics, 203.

BARNETT, I. A. Integro-Differential Equations with Constant Limits of Integration, 193.

BeLL, E. T. On the Number of Representations of $2 n$ as a Sum of $2 r$ Squares, 19.

- Definition and Illustrations of New Arithmetical Group Invariants, 211.

- The Equation $d s^{2}=d x^{2}+d y^{2}+d z^{2}, 312$.

Bennett, A. A. Poncelet Polygons in Higher Space, 274.

- An Acknowledgment of Priority, 280.

Bernstein, B. A. Reports of Meetings of the San Francisco Section: October Meeting, 152; April Meeting, 396.

Birkнoff, G. D. See Reviews, under Poincare, Whittaker.

BuIss, G. A. Some Recent Developments in the Calculus of Variations, 343.

Borden, R. F. On the Adjoint of a certain Mixed Equation, 408.

CaIrns, W. D. A Derivation of the Equation of the Normal Probability Curve, 105.

- Certain Properties of Binomial Coefficients, 160.

Carmichate, R. D. Note on Convergence Tests for Series and on Stieltjes Integration by Parts, 97.

- Note on a Physical Interpretation of Stieltjes Integrals, 102, 136.

- See Reviews, under Ahrens, Castelnuovo, de Mendizabal Tamborrel.

Carver, W. B. and King, E. F. A Property of Permutation Groups Analogous to Multiple Transitivity, 319 .

ChaO, Y. R. A Note on "Continuous Mathematical Induction," 17.

Cole, F. N. Reports of Meetings of the American Mathematical Society: October Meeting, 145; Twenty-Sixth Annual Meeting, 241; February Meeting, 337; April Meeting, 433.

Cowley, E. B. See Reviews, under Brenke, Merrill, Skinner.

Craig, C. F. See Reviews, under McClenon.

Crathorne, A. R. See Reviews, under Hancock, Running.

Currier, C. H. See Reviews, under Clements, Skinner.

Curtis, M. F. On the Rectifiability of a Twisted Cubic, 275.

Daniell, P. J. Stieltjes Derivatives, 444.

DinI, U. See Ford, W. B.

Dowling, L. W. See Reviews, under Browne. 
Dresden, A. Report of the April Meeting of the American Mathematical Society at Chicago, 385 .

Dresden, A., and Kellogg, O. D. Report of the St. Louis Meeting of the American Mathematical Society, 260.

Fond, W. B. A Brief Account of the Life and Work of the Late Professor Ulisse Dini, 173.

FonsyTH, C. H. Formulas for Constructing Abridged Mortality Tables for Decennial Ages, 34, 135.

- See Reviews, under Sutton.

GABA, M. G. A Set of Completely Independent Postulates for the Linear Order $\eta, 158$.

Green, G. M. See Wilczynski, E. J.

HaYashi, T. On the Rectifiability of a Twisted Cubic, 73, 136.

Hildebrandt, T. H. On Bordered Fredholm Determinants, 400.

Karpinski, L. C. See Reviews, under Archibald.

Kalloga, O. D. See Dresden, A.

King, E. F. See Carver, W. B.

Lehmer, D. N. See Reviews, under Dickson.

LunN, A. C. Some Functional Equations in the Theory of Relativity, 26.

MACNeIsh, H. F. The Sum of the Face Angles of certain Polyhedrons in $n$-Space, 398.

Miller, G. A. Form of the Number of Subgroups of Prime Power Groups, 66.

- Groups Generated by Two Operators of Order Three whose Product is of Order Four, 361 .

- See Reviews, under Cajori.

MrLne, W. E. Infinite Systems of Functions, 294.

Moore, C. L. E. Rotation Surfaces of Constant Curvature in Space of Four Dimensions, 454.

Moore, R. L. See Reviews, under Veblen.

Moduton, E. J. Report of the Twenty-Sixth Summer Meeting of the American Mathematical Society, 49.

Owens, F. W. See Reviews, under Dowling.

Pitcher, A. D. On the Coherence of Certain Systems in General Analysis, 405.

Reynolds, C. N., JR. Note on Linear Differential Equations of the Fourth Order whose Solutions Satisfy a Homogeneous Quadratic Identity, 277.

Richandson, R. G. D. See Reviews, under Bôcher.

SAFFord, F. H. Reduction of the Elliptio Element to the Weierstrass Form, 13.

- Parametric Equations of the Path of a Projectile when the Air Resistance Varies as the $n$th Power of the Velocity, 289.

- See Reviews, under Lepper.

Shaw, J. B. See Reviews, under Cullis. 
Slocum, S. E. See Reviews, under Taylor, Wentworth.

TAYLOR, J. S. Sheffer's Set of Five Postulates for Boolean Algebras in Terms of the Operation "Rejection" Made Completely Independent, 449.

Walsh, J. L. On the Proof of Cauchy's Integral Formula by Means of Green's Formula, 155.

Wilczynski, E. J. In Memory of Gabriel Marcus Green, 1.

WiLson, W. H. On Certain Related Functional Equations, 300.

Winger, R. M. Some Generalizations of the Satellite Theory, 75 .

\section{Review.}

Ahrens, W. Mathematische Spiele (dritte Auflage), R. D. Carmichate, 86.

Allen, F. See Taylor, E. H.

Archibald, R. C. The Training of Teachers of Mathematics for the Secondary Schools. With the Editorial Cooperation of D. E. Smith, W. F. Osgood, and J. W. A. Young, L. C. KARPINSKI, 179.

Bôcher, M. Leçons sur les Méthodes de Sturm dans la Théorie des Equations Différentielles linéaires et leur Développements modernes, recueillies et rédigées par G. Julia, R. G. D. Richardson, 108.

Brenke, W. C. Advanced Algebra, E. B. Cowley, 323.

Brown, J. C. See Wentworth, G.

Browne, R. T. The Mystery of Space, L. W. Dowling, 460.

Cajori, F. A History of Mathematics, G. A. Miller, 79.

Castelnuovo, G. Calcolo delle Probabilità, R. D. Carmichael, 132.

Clements, G. R. Problems in the Mathematical Theory of Investment, C. H. Currier, 463.

Cullis, C. E. Matrices and Determinoids, volumes 1 and 2, J. B. SHAw, 224.

Dickson, L. E. History of the Theory of Numbers, volume 1, D. N. LeHMER, 125, 281.

Dowling, L. W. Projective Geometry, F. W. Owens, 39.

Hancock, H. Theory of Maxima and Minima, A. R. Crathorne, 180.

Julia, G. See Bôcher, M.

Lennes, N. J. See Sutton, C. W.

Lepper, G. H. From Nebula to Nebula (fourth edition), F. H. SAFroRd, 182.

McClenon, R. B. and Rusk, W. J. Introduction to the Elementary Functions, C. F. Cratg, 40.

Mendizabal Tamborrel, J. de. Tratado Elemental de Goniometria (segunda edición), R. D. Carmichate, 86.

Merrill, H. A. and Smith, C. E. A First Course in Higher Algebra, E. B. CoWley, 323.

Osgood, W. F. See Archibald, R. C. 
Poincaré, H. Oeuvres, tome 2, G. D. Birkhoff, 164.

Running, T. R. Empirical Formulas, A. R. CrathorNe, 376.

Rusk, W. J. See McClenon, R. B.

Schlauch, W. S. See Wentworth, G.

Skinner, E. B. College Algebra, E. B. CowLey, 323.

- Tables from the Mathematical Theory of Investment, C. H. CorRIER, 463.

Smith, C. E. See Merrill, H. A.

Smith, D. E. See Archibald, R. C., Wentworth, G.

Sutton, C. W., and Lennes, N. J. Business Arithmetic, C. H. Forsyth, 375.

Taylor, E. H., and Allen, F. Junior High School Mathematics, books 1 and 2, S. E. SLOCUM, 462.

Veblen, O., and Young, J. W. Projective Geometry, volume 2, R. L. Moore, 412.

Wentworth, G., Smith, D. E., and Brown, J. C. Junior High School Mathematics, S. E. SLOCUM, 373.

Wentworth, G., Smith, D. E., and Schlauch, W. S. Commercial Algebra, F. E. AlLEN, 177 .

Whittaker, E. T. A Treatise on the Analytical Dynamics of Particles and Rigid Bodies; with an Introduction to the Problem of Three Bodies (second edition), G. D. BirKHoFf, 183.

Young, J. W. See Veblen, O.

Young, J. W. A. See Archibald, R. C.

Corrections, 135, 380.

Index of Volume XXVI, 480.

New Publications, 46, 94, 142, 189, 237, 285, 334, 380, 430, 469.

Notes, 41, 87, 136, 184, 233, 281, 329, 377, 425, 464.

Papers Read before the Society and Subsequently Published, TwentyNinth Annual List of, 472.

\section{Notes AND OTHER ITEMS.}

Academies, Associations, Congresses, and Societies:

American Mathematical Society: Annual Meeting, 136; Committee on Bibliography, 242, 435; Election of Officers, 243; Incorporation, 242, 434; List of Officers and Members, 136; New Members Admitted, 49, 145, 242, 337, 434; St. Louis Meeting, 136; San Francisco Section, June Meeting, 396; Statistics, 243; Summer Meeting and Colloquium, 329 ; Transactions, $87,184,234,426,434$.

Associations for the Advancement of Science, American, 136, 233, 377, 467; British, 377.

American Astronomical Society, 87; Astronomical Society of the Pacific, 283; Belgian Academy of Sciences, 138; Edinburgh Mathematical Society, 184, 331, 464; Göttingen Society of Sciences, 331; 
International Mathematical Union, 184, 337, 425, 435, 464; London Mathematical Society, 87, 330; Mathematical Association of America, 87, 136, 233, 234, 425; Mathematical Association of Japan, 282; Mathematical Society of Greece, 377; National Academy of Sciences, 137, 426; National Research Council, 184, 337, 425; Norwegian Mathematical Society, 42; Paris Academy of Sciences, 138, 282; Prince Jablonowski Society, 332; Royal Institute of Venice, 91; Royal Society of London, 185, 282; Strasbourg Congress, 330, 464.

Catalogues of Books, 188, 237, 469 .

Doctorates in Mathematics, American, 137.

Journals: American Journal of Mathematios, 42, 234, 330, 464; Annals of Mathematics, 41,136, 281, 426; Bulletin of the Mathematical Society of Greece, 377; Bulletin of the Norwegian Mathematical Society, 42; L'Education Mathématique, 138; Giornale di Matematica Finanziaria, 282; Jahrbuch über die Fortschritte der Mathematik, 235; Journal of the Mathematical Association of Japan, 282; Mathematische Annalen, 465; Mathematische Zeitschrift, 42; Proceedings of the National Academy of Sciences, 41; Revue de Mathématiques Spéciales, 138; Transactions of the American Mathematical Society, 87, 184, 234, $426,434$.

Papers and Communications Presented to the Society, Authors:

Alexander, J. W., 52, 338, 338.

Alger, P. L., 244.

Allen, E. S., 52.

Altshiller-Court, N., 262, 262 , 435.

Barnett, I. A., 435, 435 .

Bell, E. T., 152, 152, 152, 396, $396,396$.

Bennett, A. A., 146, 146, 146, 146, 244, 244, 338 .

Birkhoff, G. D., 146, 261, 436.

Blichfeldt, H. F., 396.

Bliss, G. A., 53, 53, 261.

Borden, R. F., 261.

Borger, R. L., 262.

Bouton, C. L., 245.

Boutroux, P., 262, 338.

Brink, R. W., 52.

Brown, E. W., 50.

Buchanan, D., 245.

Cairns, W. D., 53.

Cajori, F., 152, 396.

Camp, B. H., 338.

Carmichael, R. D., 53, 53, 53, 146, 146, 146, 388, 388, 388.

Carver, W. B., 245.

Coble, A. B., 262, 262, 388.

Cohen, T., 245.

Coolidge, J. L., 244.

Curtis, M. F., 245.

Curtiss, D. R., 53, 388.

Dantzig, T., 435.

Dickson, L. E., 435

Dines, L. I., 53.

Dodd, E. L., 262.
Douglas, J., 435.

Eisenhart, L. P., 53, 53, 435.

Emch, A., 262.

Ettlinger, H. J., 52, 262, 262.

Evans, G. C., 53, 262.

Field, P., 52.

Fischer, C. A., 245.

Fite, W. B., 245.

Ford, L. R., 435.

Ford, W. B., 52.

Forsyth, C. H., 245, 245.

Fort, T., 262.

Glenn, O. E., 244, 244, 388.

Graustein, W. C , 245, 245.

Gronwall, T. H., 146, 338, 338.

Hardy, G. H., 338.

Haskell, M. W., 152.

Hazlett, O. C., 146, 245.

Hildebrandt, T. H., 53.

Hollcroft, T. R., 435.

Hoskins, L. M., 396.

Howe, H. A., 53 .

Howland, L. A., 244.

Huntington, E. V., 50.

Jackson, D., 244, 388.

James, G. O., 261.

Kasner, E., 146, 338.

Kellogg, O. D., 146, 436.

Kempner, A. J., 52, 53.

Keyser, C. J., 244.

King, E. F., 245.

Kline, J. R., 389.

Lane, E. P., 52.

Lefschetz, S., 262.

Linfield, B. Z., 261. 
Lipka, J., 244, 338, 436, 436.

Love, C. E., 261.

Iunn, A. C., 262, 386.

McEwen, G. F., 262, 263.

MacMillan, W. D., 388.

MacNeish, H. F., 243.

Manning, W. A., 152, 152.

Mason, M., 386.

Mason, T. E., 388.

Miller, G. A., 52, 262, 262, 388.

Moore, C. L. E., 244, 244, 436.

Moore, E. H., 389.

Morse, H. C. M., 244.

Mullikin, A. M., 146.

Nelson, A. L., 52.

Page, L., 435.

Pell, A. J., 146.

Pfeiffer, G. A., 244.

P.illips, H. B., 244, 244.

Platt, H. H., 261.

Post, E. L., 435, 435.

Reynolds, C. N., Jr., 52, 52, 245.

Rice, L. H., 52.

Rider, P. R., 388.

Rietz, H. L., 53, 262.

Ritt, J. F., 435.
Roe, E. D., Jr., 52.

Roever, W. H., 53, 389.

Rowe, J. E., 245, 436.

Rutledge, G., 388.

Sakellariou, N., 436.

Schlesinger, F., 50.

Schmiedel, O., 261.

Schweitzer, A. R., 338, 338, 435.

Sharpe, F. R., 52.

Shaw, J. B., 53.

Shugert, S. P., 52.

Simonds, E. F., 146.

Snyder, V., 52.

Steimley, L. L., 262.

Stouffer, E. B., 388.

Taylor, J. S., 245.

Vandiver, H. S., 436.

Veblen, O., 436 .

Wahlin, G. E., 388.

Walsh, J. L., 244, 244, 245.

Wedderburn, J. H. M., 338.

Whittemore, J. K., $146,435$.

Wiener, N., 244, 244, 244, 244.

Wilczynski, E. J., 262, 388.

Williams, K. P., 262, 388.

Winger, R. M., 152 .

Zeldin, S. D., 245.

Personal Notes:

Alasia de Quesada, C., 45; Albright, B., 284; Alexander, J. W., 467; Allen, E., 235; Allen, E.'S., 140; Ames, L. D., 235; Anderegg, F., 467; Andoyer, H., 44; Anning, N., 236; Appell, P., 44, 429; Arms, R. A., 187; Atwood, O., 141.

Babbitt, A. E., 468; Baier, F. K., 467; Baire, R., 138; Baker, A., 333; Baldus, R., 185; Ballantyne, J. P., 333; Barnays, P., 185; Barnett, I. A., 430: Barney, I., 92; Barton, R. M., 235; Beal, F. W., 92; Bell, R. J. T., 283; Bennett, A. A., 140; Berwick, W. E. H., 466; Berzolari, L., 235; Bianchi, L., 379; Birkeland, 42; Birkhoff, G. D., 91, 136, 233, 329, 379, 425, 434; Blaess, V., 185; Blankenstein, E. C., 140; Blaschke, W., 42, 185; Blichfeldt, H. F., 426; Bliss, G. A., 136, 377; Blumberg, H., 45; Bôcher, M., 140; Boersch, A., 469; Böhmer, P. E., 185; Boquet, F., 38: Borden, R. F., 379; Borgmeyer, C. J., 285; Born, M., 378; Bortolotti, E., 90; Böttcher, E., 188; Boussinesq, V. J., 139; Boutroux, P., 91; Boyd, P. P., 283; Bradshaw, J. W., 140; Bragg, W. H., 466, 466; Brahana, H. R., 430; Bramble, C. C., 186; Brand, L., 137; Brandenberger, C., 45; Brewster, J. A., 333; Brink, R. W., 467; Bromwich, T. J. I., 87: Brouwer, L. E., 465; Brown, E. W., 283; Brown, F. L., 187; Bruce, R. E., 283; Brunn, A. von, 378; Burchnall, L. L., 283; Bussey, W. H., 467; Butterfield, A. D., 380.

Cairns, W. D., 467; Camp, C. C., 284; Campbell, A. D., 92; Campbell, J. E., 87: Campbell, J. W., 187; Cantor, M., 380, 465; Carathéodory, C., 465; Carmichael, R. D., 234; Carpenter, A. F., 185; Castelnuovo, G., 90; Chapelon, 139; Chapman, F. E., 93; Chapman, S., 90; Charbonnier, P., 138; Charles, R. L., 187; Chazy, J., 44; Ćlairin, J. 44; Collet, 139; Collier, M., 140; Colpitts, M. A., 93; Conwell, H. H., 187; Cowan, E. E., 430; Cresse, G. H., 137; Crocco, 
A., 235; Cummings, L. D., 141; Curtis, H. B., 187; Curtiss, D. R., 233, 377, 429; Czuber, E., 185.

Daniele, E., 235; Daniells, M. E., 187; Dantzig, T., 93; Danzer, O., 188; Davis, H. N., 140; Davis, J. E., 284; Dedekind, J. W. R., 235; De Donder, T., 184, 282; De Lury, A. T., 333; Demartres, G., 188; Demoulin, A., 138; Denjoy, A., 44; De Porte, J. V., 187; Dickson, L. E., 425, 430, 464; Dotterer, J. E., 284; Douglas, J., 468; Dresden, A., 186; Dueker, O. W., 284; Dumont, E., 45; Dunkel, O., 141; Duval, E. P. R., 430; Dziobek, O., 188.

Eddington, A. S., 138, 377; Ehrman, C. D., 284; Einstein, A., 332, 467; Eisenhart, L. P., 434, 464; Emch, A., 91; Epstein, P., 185; Everett, J. R., 468.

Fairon, J., 139; Falckenberg, H., 378; Fehr, H., 43; Feltges, E. M. F., 284; Feussner, W., 43; Field, P., 140; Fisher, G. E., 380; Flamme, D. J. B., 466; Flexner, S., 233; Floquet, A. M. G., 139; Forsyth, C. H., 186; Fraleigh, P. A., 284; Frege, G., 43; Furukawa, H., 282.

Garnier, R., 466; Garretson, W. V. N., 92; Gau, E., 139; Geer, P. van, 468; Gibbens, G., 467; Giraud, G., 138, 466; Glover, J. W., 91; Gob, A., 188; Godeaux, L., 138; Gossard, H. C., 186; Got, L. A. T., 139; Gouwnes, C., 468; Graber, M. E., 45; Graefe, F., 188; Gronwall, T. H., 91; Gross, W., 45; Grove,V. G., 284; Grüneisen, E., 43; Guichard, C., 44; Guimaraes, R., 188.

Haag, J., 139; Hadamard, J., 332, 379, 466; Hadley, L., 186; Haid, M., 468; Hardy, G. H., 87, 235; Harris, R. A., 140; Harrison, W. J., 332; Hart, W. L., 467; Hassé, H. R., 44; Hayashi, T., 282; Hazlett, O. C., 467; Heath, T. L., 466; Hebbert, C. M., 187; Hecke, E., 185; Hedrick, E. R., 234; Heger, R., 469; Heinz, A. H., 283; Hemke, P., 187; Herdman, W. A., 377; Hess, G. W., 468; Hessenberg, G., 43, 139; Hilbert, D., 332; Ho, Y. H., 333; Hodge, F. H., 186; Holder, F. J., 141; Holgate, T. F., 140; Howe, A. M., 468; Humbert, P., 44; Huntington, E. V., 44, 91, 377; Hurwitz, A., 236; Husson, A. S. E., 466.

Ingraham, M., 284.

Jackson, D., 377; Jackson, T. W., 236; Jacobs, J. M., 137; Jacobsthal, E., 43; James, G., 186; James, G. D., 236; Janet, 139; Jeans, J. H., 185, 282; Johnson, M. F., 140; Jones, J. L., 333; Jordan, C., 426; Jourdain, P. E. B., 188; Jung, H., 466.

Kaba, M., 282; Kampé de Fériet, J., 139; Karpinski, L. C., 140; Kazarinoff, D. C., 140; Keller, J., 45; Kellogg, O. D., 233, 379; Kells, L. M., 93; Kent, F. C., 236; Kerr, F. L., 187; Killing, W., 139; Kircher, E. A. T., 92; Klein, F., 42, 43; Kline, J. R., 91; Knebelman, M. S., 93; Knopp, K., 185; Koenigs, G., 184; Kostka, C., 42; Krathwohl, W. C., 141; Krause, J. M., 185, 378; Kuroda, M., 282; Küstermann, W. W., 91 .

Lamb, H., 466; Landis, W. W., 333; Lane, E. P., 92; Langman, H., 187; Larmor, J., 379; Lattès, S., 44; Laue, M. von, 378; Laura, E., 90; Leau, L., 139, 466; Lebesgue, H., 44, 138; Lefschetz, S., 282, 
430, 466; LeStourgeon, F. E., 93; Levi, F., 185; Levi-Civita, T., 90; Lewis, F. P., 186; Lietzmann, W., 42; Lipka, J., 44; Loewy, A., 139; Longley, W. R., 283; Lorey, W., 43; Luck, J. J., 186; Lufkin, F. M., 284.

McCain, G. I., 137; McClenon, R. B., 186; McKelvey, J. V., 93, 430; McMahon, J., 333; Maclaurin, R. C., 237; Maclay, J., 188; MacLean, N. B., 283; MacMahon, P. A., 185; MacMillan, W. D., 140; MacNeish, H. F., 187; Maddox, A. C., 92; Malstroem, R., 469; Mangoldt, $H$. von, 43; Manning, $H$. P., 379; Mansion, P., 45; Mason, T. E., 186; Mathews, R. M., 141; Meacham, E. D., 186; Meek, H. B., 468; Merlin, E. L. A., 139; Merrill, H. A., 234; Miller, G. A., 44, 377; Miller, J. S., 284; Miller, K. A., 92; Millikan, R. A., 466; Millosewich, E., 236; Mills, C. N., 186; Milne, W. E., 141; Miser, W. L., 141; Mises, R. von, 185, 465; Mohrmann, A., 43; Monroe, L. M., 92; Morse, D. S., 333; Morse, H. C. M., 284; Moulton, F. R., 44, 233, 329, 377; Muir, T., 429; Musselman, J. R., 141,468 .

Nagao, S., 282; Nelson, C. A., 430; Netto, E., 188; Noble, C. A., 141; Noether, E., 185; Nörlund, N. E., 43.

Olbrich, W., 378; Olson, H. L., 236.

Page, J. M., 377; Palmström, A., 42, 379; Parsons, F. W., 187; Pellet, A. C. E., 466; Perron, O., 332; Peterson, O. J., 140; Petrowitch, N., 184; Phalen, H. R., 92; Pierce, T. A., 92; Pincherle, S., 90; Pitcher, A. D., 377; Plemelj, J., 282; Pochhammer, L., 332; Poincaré, L., 429; Pollard, S., 379; Poritsky, H., 333; Pounder, I. R., 333; Pratt, A. S., 333; Proudman, J., 235; Putnam, T. M., 91.

Radon, J., 185; Raiford, T. S., 236; Ramanujan, S., 468; Ramsey, A., 284; Rankin, W. W., 45; Ranum, A., 333; Rayleigh, Lord, 45, 332; Rechart, F. E., 284; Reed, F. W., 92; Reina, V., 184, 236; Reye, K. T., 188; Reynolds, C. N., 137, 430; Rice, L. H., 236; Rietz, H. L., 377; Robison, G. M., 92; Roever, W. H., 377; Roman, I. A., 93; Rowe, J. E., 379, 467; Roy, L., 44.

Sakellariou, N., 377; Schmeidler, M., 185; Schmidt, E., 43; Schur, F., 42, 139; Schur, I., 332, 465; Sensenig, W., 137; Servais, C., 282; Sharpe, F. R., 333; Sherk, W. H., 236; Sherwood, G. E. F., 140; Shively, L. S., 236; Shugert, S. P., 93; Shumway, R. R., 141; Simon, W. G., 430; Simpson, T. McN., 236; Sire, L., 466; Slaught, H. E., 234; Smith, D. E., 234; Smith, H. L., 93; Smith, M. G., 92; Smith, P. F., 434; Snow, C., 380; Snyder, V., 44; Solberg, 42; Sousley, C. P., 284; Sperry, M. J., 468; Stäckel, P., 469; Stebbins, J., 377; Stephens, R. P., 141; Stickelberger, L., 139; Störmer, F. C. M., 42; Strutt, J. W., 45, 332; Sturm, R., 188; Stuyvaert, M. L. M., 139; Suppantschitsch, R., 282.

Tanzola, J. J., 188; Tauber, A., 379; Taylor, E., 93; Taylor, J. M., 141; Thomson, J. J., 235, 283; Tietze, H., 139; Timerding, H. E., 43; Timpe, A., 43; Torrey, M. M., 468; Torroja, E., 93; Trayler, I. W., 284 ; Trefftz, E., 378; Tripp, M. O., 45; Trowbridge, A., 466; Tsoo, C. C., 137; Turrière, E. L. F., 139.

Underhill, A. L., 467; Underwood, R. S., 236; Upton, C. B., 235. 
Vallée Poussin, C. de la, 184; Vance, E. H., 91; Vandiver, H. S., 92; Van Vleck, E. B., 333; Veblen, O., 425; 434; Vessiot, E., 44; Voigt, W., 380; Volterra, V., 90.

Wangerin, A., 332; Watanabe, M., 282; Watson, G. N., 283; Webster, A. G., 235; Wedderburn, J. H. M., 91; Weld, L. G., 237, 261; Wells, M. E., 141; Wellstein, J., 188; Wester, C. W., 467; White, H. S., 425; Whittemore, J. K., 283; Whyte, W. A., 333; Wieleitner, H., 332; Wilczynski, E. J., 234; Williams, K. P., 283; Williams, W. L. G., 333, 379; Wilson, N. R., 283; Wilson, W.' H., 284; Wolfe, C. L. E., 137; Wolfe, H. E., 137, 235; Wolff, H. C., 91; Wood, F., 187; Woods, B. M., 186; Woodward, R. S., 467.

Yeaton, C. H., 141; Young, J. W., 234; Young, W. H., 90.

Zeldin, S. D., 93; Zeuthen, H. G., 284.

Prizes:

Astronomical Society of the Pacific, 283; Barnard Medal, 468; Belgian Academy of Sciences, 138; Belgian Government, 138; Göttingen Society of Sciences, 331; Nobel, 466; Paris Academy of Sciences, 138, 282; Peter Wilhelm Müller Foundation, 332; Prince Jablonowski Society, 332; Querini-Stampalia, 91; Royal Society of London, 185; Smith, 379.

Universities and Technical Schools.

Bologna, 87.

California, 140, 428.

Catania, 88.

Chicago, 331, 380.

Columbia, 378, 426 .

Cornell, 140, 427.

Genoa, 88.

Harvard, 184, 427.

Massachusetts Institute of Technology, 428.

Messina, 88.

Naples, 88.
Padua, 88.

Palermo, 89.

Pavia 89.

Pisa, 89.

Princeton, 465.

Rome, 89 .

Stanford, 465.

Strasbourg, 137, 429.

Turin, 90 .

Wisconsin, 428.

Yale, 379. 